\title{
ATIVIDADE TERAPÊUTICA DO ANTICORPO MONOCLONAL EVOLOCUMAB CONTRA PROTEÍNAS PCSK9 NA MODULAÇÃO DO COLESTEROL LDL: REVISÃO SISTEMÁTICA
}

\author{
THERAPEUTIC ACTION OF THE EVOLOCUMAB MONOCLONAL ANTIBODY AGAINST PCSK9 \\ PROTEINS IN LDL CHOLESTEROL MODULATION: SYSTEMATIC REVIEW
}

\section{Mario Henrique Quim Ferreira ${ }^{a}$, Rodrigo Metzker Pereira Ribeiro ${ }^{b^{*}}$, Ana Beatriz Antunes Funes ${ }^{c^{*}}$, Bruna Mohine Oliveira Faustino ${ }^{\mathrm{d}^{*}}$, Fernanda Cardin Segato ${ }^{\mathrm{e}^{*}}$, Luana Lozano Cardoso de Mattos ${ }^{\mathrm{f}^{*}}$, Thais Caroline dos Santos Carvalho ${ }^{\mathrm{g}^{*}}$, Carlos Funes Prada Filho ${ }^{\mathrm{h} * *}$}

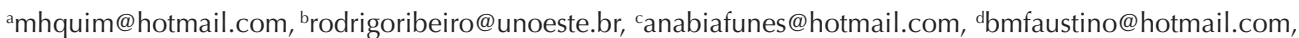
efernandasegato@outlook.com, fluanalozano@hotmail.com, sthais_thaty12@hotmail.com, hcarlinhosprada@hotmail.com *Universidade Oeste Paulista - Presidente Prudente (SP), Brasil

**Instituto Dante Pazzanese de Cardiologia - São Paulo (SP), Brasil

Data de recebimento do artigo: 07/01/2017 Data de aceite do artigo: 19/04/2017

\section{RESUMO}

Introduçáo: As alteraçôes de hipercolesterolemia podem gerar patologias como o infarto agudo do miocárdio (IAM). No entanto, nem sempre o aumento do colesterol é ocasionado apenas pela má alimentação e pelo estilo de vida; grupos como o da hipercolesterolemia familiar (HF) têm valores séricos aumentados por alteraçôes genéticas e, nesses casos, as medicaçôes antidislipidemiantes tradicionais não são eficazes. Objetivo: Compilar e discutir os resultados obtidos pela literatura atual que se debruça sobre o anticorpo monoclonal evolocumab para diminuição do PCSK9. Materiais e métodos: Revisão sistemática dos resultados de testes feitos em humanos com idade superior a 19 anos publicados em língua inglesa há menos de cinco anos, utilizando a base de dados PubMed. Resultados: Foram encontrados 135 artigos, dentre os quais nove foram selecionados por critérios de exclusão. Discussáo: Os resultados demonstraram que o poder farmacológico do anticorpo evolocumab tem notória valia diante de terapêuticas habituais e placebos para pacientes que não apresentam resposta significativa com as terapias atuais. Conclusão: Por se tratar de um estudo bastante recente, ainda há necessidade de mais pesquisas que demonstrem eventuais reaçôes adversas e interaçôes com outros medicamentos. No entanto, já está em evidência uma grande vantagem para tratamentos futuros por preencher lacunas da farmacologia tradicional.

Palavras-chave: Hiperlipoproteinemia tipo II; lipoproteínas LDL; infarto do miocárdio; PCSK9.

\section{ABSTRACT}

Introduction: The hypercholesterolemia can lead to conditions such as acute myocardial infarction (AMI). However, cholesterol elevation is not always caused by poor diet habits and lifestyle; groups such as familial hypercholesterolemia (HF) have serum values increased by genetic alterations, and traditional dyslipidemic medications are not effective enough for these peoples. Objective: To compile and discuss the results obtained by the current literature that focuses on the evolocumab monoclonal antibody to decrease PCSK9 levels. Materials and methods: Systematic review of the results of tests done with humans over 19 years of age, published in the last 5 years in the English language, using the PubMed database. Results: 135 articles were found, and nine studies were selected by exclusion criteria. Discussion: Results demonstrated that the pharmacological power of the evolocumab antibody has a remarkable value compared to the usual therapies and placebo for patients who did not show great response to current therapies. Conclusion: This is a very recent study, which requires further research to show possible adverse reactions and interactions with other drugs. However, so far it has demonstrated a great advantage for future treatments because it occupies a place not previously filled by traditional pharmacology.

Keywords: Hyperlipoproteinemia type II; LDL lipoproteins; myocardial infarction; PCSK9. 


\section{Introdução}

A ocorrência crescente de hipertensão arterial sistêmica (HAS) ${ }^{1}$, infarto agudo do miocárdio (IAM) e outras doenças relacionadas ao aumento dos valores de lipoproteína de baixa densidade $(\mathrm{LDL})^{2}$ tornou-se uma realidade rotineira da clínica médica ${ }^{3}$. Essa situação é proporcional à ascendência dos níveis dislipidêmicos na população, gerados pela má alimentação, estilo de vida inadequado ou problemas hereditários na depuração do colesterol $^{4}$, e configura-se em notória gravidade perante a saúde pública ${ }^{5}$. Como consequência e paralelamente a essa realidade, há a busca permanente por fármacos ou terapias resolutivas para a enfermidade e prevenção. Esse anseio é proveniente tanto da vontade humana, que conduz à cura, como da disputa comercial atrelada à concorrência da indústria farmacêutica ${ }^{6}$.

Existem diversas classes de medicamentos que agem em vários segmentos corporais para o combate à dislipidemia. Entre os mais utilizados na prática clínica estão a estatina $^{7,8}$, a ezetimiba ${ }^{9}$ e as resinas ${ }^{10}$. Contudo, em um cenário em que fármacos atuais atingiram seu limiar de tratamento, a comunidade científica vislumbra uma nova classe de medicamentos atuantes contra a pró-proteína convertase subtilisina/quexina tipo 9 (PCSK9 $)^{11}$ : os anticorpos monoclonais, como o evolocumab (AMG 145). Os resultados de sua utilização respaldam em torno dos receptores de lipoproteína de baixa densidade (LDLR) $)^{12}$, o que altera o fluxo da limpeza de LDL do plasma provocando diminuição da concentração sérica ${ }^{13}$.

Essa nova classe terapêutica deve auxiliar a conduta atual para os grupos de dislipidêmicos com alteração adquirida ou hereditária ${ }^{14}$. A primeira situação diz respeito aos portadores de dislipidemia gerada pelo sedentarismo e pela alimentaçáo, para os quais se busca o uso de estatinas com outros antidislipidemiantes, quando necessário, sendo essa classe de fármaco um novo auxiliar. Já o grupo dos portadores de hipercolesterolemia familiar (HF), que abarca cerca de dez milhóes de pessoas no mundo e entre $250 \mathrm{mil}$ e 300 mil no Brasil ${ }^{15}$, é composto por indivíduos que não conseguem diminuir seus níveis lipêmicos com as terapias atuais, o que acaba gerando lesóes obstrutivas precoces e aumento do risco de morte relativo à doença coronária ${ }^{16}$.

Este trabalho apresenta e discute, de forma atualizada, a atividade do fármaco evolocumab contra a enzima PCSK9, comparando sua eficácia contra placebo e fármacos existentes que se destinam a benefícios semelhantes, para assim mensurar sua importância na prática clínica futura.

\section{Função da enzima PCSK9}

A PCSK9 é uma enzima sintetizada pela expressão de um gene de mesmo nome localizado no cromossomo 1p32.3. Esse gene é altamente ativado pelo fígado, mas também pelo intestino e rins ${ }^{17}$, com a função de inibir os LDLR, classificado como o nono integrante da família das pró-proteínas convertases ${ }^{18}$.

Uma das funções dessas pró-proteínas é a ativação de proteínas transmembranais através da hidrólise após a clivagem de pares de resíduos de aminoácidos básicos Lys(Arg) $/ \operatorname{Arg}(\text { Lys })^{19}$. A interação da enzima PCSK9 se dá com o lisossoma, degradando o LDLR para evitar que essa seja reciclada para a membrana celular. Dessa maneira, ela influencia diretamente nos níveis plasmáticos de $\mathrm{LDL}^{20,21}$, o que pode gerar maiores riscos para doenças cardiovasculares (DCV).

Os LDLR se apresentam na membrana de células, como hepatócitos $(75 \%)$ ou tecidos extra-hepáticos, e são capazes de invaginar as partículas de colesterol, iniciando o processo de clearance dessa substância ${ }^{22,23}$, conferindo-lhes uma meia-vida que dura de dois a três dias $^{24-27}$. Estudos apontam que $90 \%$ da regulação plasmática de LDL se deve à função dos receptores transmembranares dessa lipoproteína ${ }^{28}$.

Segundo Cohen et al. ${ }^{29}$, em trabalhos realizados com pessoas com decréscimo natural da expressão do gene PCSK9, comprovou-se a diminuição conjunta dos níveis séricos de colesterol de LDL, concluindo, por meio desta observação, que medicamentos que atuam diminuindo a expressão dessa enzima aumentam o número de LDLR e auxiliam a queda de níveis séricos de colesterol.

\section{Metodologia}

Para esta pesquisa, utilizou-se do modelo de trabalho de revisão sistemática, buscando sintetizar e discutir de maneira global os resultados recentes da literatura em torno da redução dos valores de PCSK9. Realizaram-se buscas em outubro e novembro de 2016 na base de dados PubMed. O processo de busca foi realizado de acordo com os indexados pelo DeCS (Descritores em Ciências da Saúde); contudo, apenas o termo "antibody" demonstrou resultado. Os termos "PCSK9" e "evolocumab" não foram fornecidos pelo descritor, mas foram incluídos devido a sua importância para esta pesquisa. O cruzamento dos termos foi realizado pelo operador "AND".

Quanto ao critério de seleção dos artigos encontrados, utilizaram-se somente aqueles que: 1 - forneciam texto completo de forma gratuita; 2 - trabalhos escritos em língua inglesa; 3 - artigos publicados com menos de cinco anos; 4 - feitos em humanos; 5 - em adultos maiores de 19 anos. Embora essa tenha sido a idade especificada para a seleção, alguns artigos da pesquisa que traziam valores de 18 anos foram incluídos para agregar maior amplitude às comparaçôes. 
Para confrontar os dados, utilizaram-se artigos que seguem uma das principais linhas de combate ao PCSK9 descritas na literatura atual: o anticorpo monoclonal evolocumab (AMG 145). Para garantir maior homogeneidade entre os resultados, utilizamos na pesquisa aqueles que apresentavam metodologia de dose de evolocumab de $140 \mathrm{mg}$ quinzenalmente (Q2W) e 420 mg mensalmente (QM), haja vista que outros trabalhos indicavam doses não semelhantes entre si, o que poderia alterar o cruzamento dos resultados.

O fluxograma a seguir demonstra as buscas e os respectivos resultados encontrados.

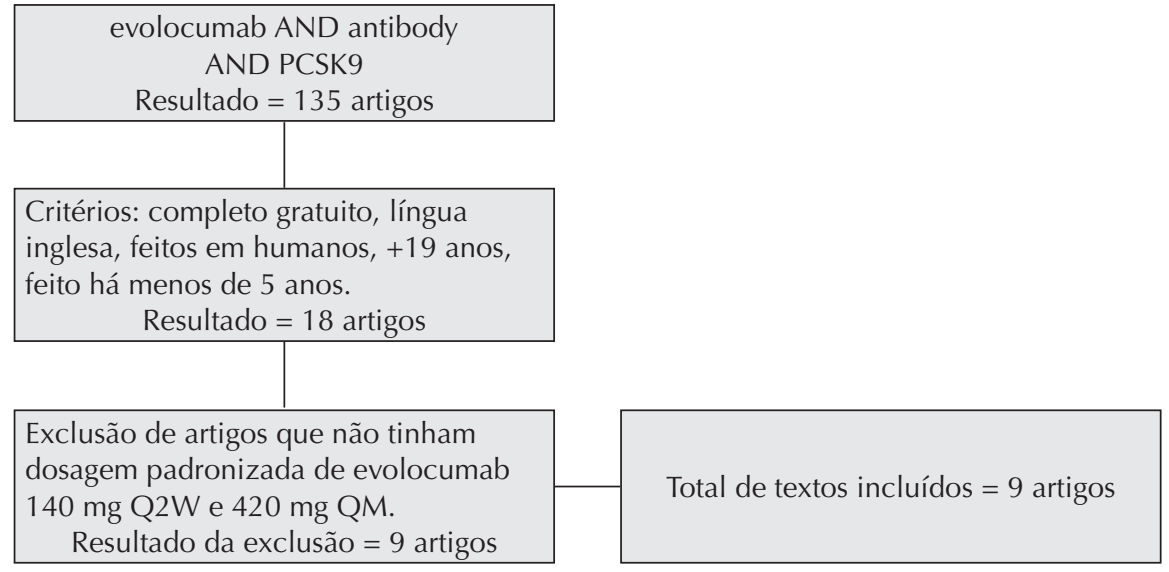

Figura 1: fluxograma para seleção dos artigos desta revisão.

\section{Resultados}

A Tabela 1 demonstra os resultados obtidos nos artigos que foram selecionados nesta revisão.

\section{Discussão}

Tomando por base os fármacos antidislipidêmicos disponíveis e o seu campo de ação por meio de suas farmacocinéticas, é possível verificar que os inibidores de PCSK9 ocupam uma vertente previamente não preenchida por outras terapias disponíveis no mercado atual, o que pode gerar benefícios inéditos, tanto na monoterapia como em associação a algum medicamento já estabelecido. Assim, sua prescrição pode preencher lacunas exclusivas, como no caso já citado de hipercolesterolemia familiar hereditária, ou em terapia somada à dislipidemia comum.

De modo absoluto, todos os artigos que demonstravam o uso de evolocumab, independente se a dosagem seguia os critérios estipulados por este estudo, apresentaram diminuição significativa nos valores de LDL-C. Essa redução se mostrou vantajosa em pacientes que utilizaram placebo, bem como em pacientes que fizeram uso de antidislipidemiantes.

Tabela 1: Demonstração dos resultados obtidos no estudo.

\begin{tabular}{|c|c|c|}
\hline Referência & Amostra e metodologia & Resultados de LDL obtidos \\
\hline $\begin{array}{l}\text { Giugliano } \\
\text { et al. }{ }^{30}\end{array}$ & $\begin{array}{l}\text { - Idade: } 18 \text { a } 80 \text { anos } \\
\text { - Amostra: } 631 \text { pacientes } \\
\text { - Tempo de pesquisa: } 12 \text { semanas } \\
\text { - Metodologia: Q2W com doses de } 70 \mathrm{mg}, 105 \mathrm{mg} \text {, ou } 140 \mathrm{mg} \text { de AMG } 145 \text { ou } \\
\text { placebo, e QM com doses } 280 \mathrm{mg}, 350 \mathrm{mg} \text {, ou } 420 \mathrm{mg} \text {, ou placebo }\end{array}$ & $\begin{array}{l}\text { Concentraçóes de LDL reduziram de } \\
\text { maneira diferente, dependendo da dose, } \\
\text { variando de } 41,8 \text { a } 66,1 \% \text { para Q2W e } \\
41,8 \text { a } 50,3 \% \text { para QM. }\end{array}$ \\
\hline $\begin{array}{l}\text { Kohli } \\
\text { et al. }{ }^{31}\end{array}$ & $\begin{array}{l}\text { - Idade: } 18 \text { a } 80 \text { anos } \\
\text { - Amostra: } 629 \text { pacientes } \\
\text { - Tempo de pesquisa: } 12 \text { semanas } \\
\text { - Metodologia: diferentes doses de AMG } 145 \text { Q2W e QM comparadas com placebo }\end{array}$ & $\begin{array}{l}\text { Os grupos demonstraram resultados com } \\
\text { potencial de } 99 \% \text { para ilustrar uma reduçáo } \\
\text { de LDL-C de cerca de } 20 \% \text { entre AMG- } \\
145 \text { e o placebo. }\end{array}$ \\
\hline $\begin{array}{l}\text { Cho } \\
\text { et al. }{ }^{32}\end{array}$ & $\begin{array}{l}\text { - Idade: } 18 \text { a } 80 \text { anos } \\
\text { - Amostra: } 300 \text { pacientes } \\
\text { - Tempo da pesquisa: } 12 \text { semanas } \\
\text { - Metodologia: comparaçáo entre os grupos placebo + evolocumab e placebo + } \\
\text { ezetimiba }\end{array}$ & $\begin{array}{l}\text { Este estudo mostrou uma variaçáo de } 40 \% \\
\text { a } 60 \% \text { de LDL-C com } 94 \% \text { dos pacientes } \\
\text { apresentando mínimo efeito colateral. }\end{array}$ \\
\hline
\end{tabular}

continua.. 


\begin{tabular}{|c|c|c|}
\hline Referência & Amostra e metodologia & Resultados de LDL obtidos \\
\hline $\begin{array}{l}\text { Hirayama } \\
\text { et al. }{ }^{33}\end{array}$ & $\begin{array}{l}\text { - Idade: } 20 \text { a } 85 \text { anos } \\
\text { - Amostra: } 310 \text { pacientes } \\
\text { - Tempo de pesquisa: } 12 \text { semanas } \\
\text { - Metodologia: comparaçáo dos grupos Q2W evolocumab e placebo e grupos QM } \\
\text { evolocumab e placebo }\end{array}$ & $\begin{array}{l}\text { O percentual de diminuiçáo do grupo } \\
\text { Q2W versus placebo foi de } 74,9 \% \text {; do } \\
\text { grupo QM, com relação ao placebo, foi de } \\
70,9 \% \text {. }\end{array}$ \\
\hline $\begin{array}{l}\text { Koren } \\
\text { et al. }{ }^{34}\end{array}$ & $\begin{array}{l}\text { - Idade: } 18 \text { a } 80 \text { anos } \\
\text { - Amostra: } 614 \text { pacientes } \\
\text { - Tempo da pesquisa: } 12 \text { semanas } \\
\text { - Metodologia: comparação de ezetimiba, placebo e evolocumab }\end{array}$ & $\begin{array}{l}\text { O tratamento com evolocumab reduziu o } \\
\text { LDL-C, em média, } 55 \% \text { a } 57 \% \text { em relação } \\
\text { ao placebo e } 38 \% \text { a } 40 \% \text { em relação ao } \\
\text { ezetimiba. }\end{array}$ \\
\hline $\begin{array}{l}\text { Stein } \\
\text { et al. }{ }^{35}\end{array}$ & $\begin{array}{l}\text { - Idade: } 56,4 \pm 11,7 \\
\text { - Amostra: } 1359 \text { pacientes } \\
\text { - Tempo de pesquisa: } 12 \text { semanas } \\
\text { - Metodologia: uso de ezetimiba, evolocumab ou placebo nas fraçóes Q2W ou QM }\end{array}$ & $\begin{array}{l}\text { As médias de reduçáo entre os grupos Q2W } \\
\text { e QM de placebo e evolocumab foram de } \\
40,2 \% \text { e } 59,3 \% \text {, respectivamente. }\end{array}$ \\
\hline $\begin{array}{l}\text { Stroes } \\
\text { et al. }{ }^{36}\end{array}$ & $\begin{array}{l}\text { - Idade: } 18 \text { a } 80 \text { anos } \\
\text { - Amostra: } 307 \text { pacientes } \\
\text { - Tempo da pesquisa: } 12 \text { semanas } \\
\text { - Metodologia: comparaçáo do tratamento com evolocumab e ezetimiba }\end{array}$ & $\begin{array}{l}\text { A redução média no Q2W foi de } 56,1 \% \text {, e } \\
\text { o de QM foi de } 55,3 \% \text { em relação à linha } \\
\text { de base. Já o ezetimba produziu reduçóes de } \\
36,9 \% \text { e } 38,7 \% \text {, respectivamente. }\end{array}$ \\
\hline $\begin{array}{l}\text { Sabatine } \\
\text { et al. } .^{37}\end{array}$ & $\begin{array}{l}\text { - Idade: grupo evolocumab } 57,8 \pm 11 \text {, e grupo terapia padrão } 58,2 \pm 10,9 \\
\text { - Amostra: } 4465 \text { pacientes } \\
\text { - Tempo da pesquisa: } 12 \text { semanas } \\
\text { - Metodologia: evolocumab + terapia padrão e terapia padrão }\end{array}$ & $\begin{array}{l}\mathrm{Na} 12^{a} \text { semana, a terapia de evolocumab } \\
\text { reduziu os níveis de colesterol LDL em } \\
61 \% \text {, quando comparada à terapia padrão. }\end{array}$ \\
\hline $\begin{array}{l}\text { Kiyosue } \\
\text { et al. }{ }^{38}\end{array}$ & $\begin{array}{l}\text { - Idade: } 20 \text { a } 85 \text { anos } \\
\text { - Amostra: } 409 \text { pacientes } \\
\text { - Tempo da pesquisa: } 12 \text { semanas } \\
\text { - Metodologia: grupos de atorvastatina + placebo ou atorvastatina + evolocumab }\end{array}$ & $\begin{array}{l}\text { Pacientes que receberam evolocumab + } \\
\text { atorvastatina tiveram uma redução de LDL } \\
\text { de } 67 \% \text { a } 76 \% \text { em relação àqueles que } \\
\text { receberam atorvastatina + placebo. }\end{array}$ \\
\hline
\end{tabular}

Alguns trabalhos que fizeram pesquisas de colesterol total, valores de HDL e lipoproteína A - LP(a) - também evidenciaram vantagens para o paciente, constatando uma diminuição do colesterol em geral e da LP(a) e aumento de HDL. Os mecanismos geradores desses efeitos secundários não foram completamente elucidados pelos respectivos autores.

A diferença de idade dos voluntários dos estudos esclarece a abrangência desse tratamento, sendo indicado, de acordo com os resultados, para todos os níveis etários de pessoas, a partir dos 18 anos, acometidas pela hipercolesterolemia. Quanto ao período de administração da dose ser quinzenal ou mensal, os estudos apresentaram pequena divergência nos resultados, alguns indicando melhor resposta terapêutica à dosagem Q2W e outros, à QM.

As situações em que as reduções de valores lipêmicos com evolocumab foram maiores do que as terapias com atorvastatina enfatizam a existência da fisiopatologia da hipercolesterolemia familiar hereditária, sendo esta de difícil ou nenhuma aceitação com a farmacologia disponível no mercado atual, mas que pode ser tratada por inibidores de PCSK9, devido às teorias de formação da HF.

Os efeitos colaterais vistos nas pesquisas foram em geral de pequeno agravo, com percentual homogêneo entre o grupo tratado com evolocumab e o seu comparativo. Os de maior importância foram percebidos em situaçóes esparsas, como aumento de ALT/AST em 1\% da amostra e aumento de $\mathrm{CK}>5 \mathrm{em} 0,5 \%{ }^{38}$. Esses valores indicam que esse gênero de drogas pode ser uma via de tratamento seguro para o futuro ${ }^{36}$, tendo por base os bons resultados concomitantes e os diminutos efeitos adversos.

\section{Considerações finais}

Devido ao até então baixo número de pesquisas, não é possível mensurar, de maneira consistente, a amplitude ou dimensão dessa nova terapêutica, por ainda não ser possível mesclar os resultados e compor um tratamento estatístico que compare os medicamentos disponíveis hoje no mercado. Contudo, com o que já se pode observar, é plausível afirmar que, em geral, os dados convergem para respostas positivas quanto à diminuição dos níveis de LDL, o que vai ao encontro do papel principal desta pesquisa.

Mediante a comprovação da legitimidade desse novo caminho de tratamento, as respostas trarão um novo leque de condutas para uma sobrevida de qualidade a uma sociedade que cada vez mais é afligida pelas doenças crônicas não transmissíveis, sejam essas passíveis de mudança com o estilo de vida ou não, como os casos de HF. Entretanto, independente da causa, é evidente que a ciência contemporânea busca evoluçáo constante e estabelece compromisso permanente com as soluções da saúde humana.

\section{Referências}

1. Marte AP, Santos RD. Bases fisiopatológicas da dislipidemia e hipertensão arterial. Rev Bras Hipertens. 2007;14(4):252-7.

2. Duarte M, Moresco RN, Bem AFD. Metodologia para a determinação da LDL oxidada e sua aplicação como marcador de risco cardiovascular. Rev Bras Anal Clin. 2008;40(2):101-6. 
3. Perez M, José D. Complicaçóes cirúrgicas do infarto agudo do miocárdio: uma revisão da literatura. XXVII Jornada Científica Internato; 2016 28-30 Out; Teresópolis, Rio de Janeiro, Brasil. Teresópolis: Unifeso; 2016.

4. Péres DS, Magna JM, Viana LA. Portador de hipertensão arterial: atitudes, crenças, percepçóes, pensamentos e práticas. Rev Saúde Públ. 2003;37(5):635-42.

5. Lopes HF, Barreto Filho JAS, Riccio GMG. Tratamento não-medicamentoso da hipertensão arterial. Rev Soc Cardiol Estado São Paulo. 2003;13(1):148-55.

6. Molinari GJDP, Moreira PCS, Conterno LO. A influência das estratégias promocionais das indústrias farmacêuticas sobre o receituário médico na Faculdade de Medicina de Marília: uma visão ética. Rev Bras Educ Med. 2005;29(2):110-8.

7. Bortolotto LA. Hipertensão arterial e insuficiência renal crônica. Rev Bras Hipertens. 2008;15(3):152-5.

8. Xavier HT, Izar MC, Faria Neto JR, Assad MH, Rocha VZ, Sposito AC, et al. V Diretriz brasileira de dislipidemias e prevenção da aterosclerose. Arq Bras Cardiol. 2013;101(4):1-20.

9. Schulz I. Tratamento das dislipidemias: como e quando indicar a combinação de medicamentos hipolipemiantes. Arq Bras Endocrinol Metab. 2006;50(2):344-59.

10. Girardi JM, Girardi FA, Peters VM. Endotélio vascular e efeitos das estatinas. HU Rev. 2006;32(1):21-5.

11. Adams-Sánchez CD, Tobón-García GJ. Monoclonal antibody therapy in Cardiology and Internal Medicine. Rev Colomb Cardiol. 2016;23(4):293-300.

12. Corral P. De volta ao básico: PCSK9 como um novo alvo para o receptor LDL. Arq Bras Cardiol. 2014;102(1):5-8.

13. Torrinha JMDQ, Fleming L. Inibidores PCSK9 [tese]. Porto: Universidade Fernando Pessoa; 2015.

14. Hopkins PN, Defesche J, Fouchier SW, Bruckert E, Luc G, Cariou B, et al. Characterization of autosomal dominant hypercholesterolemia caused by PCSK9 gain of function mutations and its specific treatment with alirocumab, a PCSK9 monoclonal antibody. Circ Cardiovasc Genet. 2015;8(6):823-31.

15. Pereira AC, Gagliardi ACM, Lottenberg AM, Chacra APM, Faludi AA, Sposito AC, et al. I Diretriz brasileira de hipercolesterolemia familiar (HF). Arq Bras Cardiol. 2012;99(2):1-28.

16. Pereira C, Miname M, Makdisse M, Filho RK, Santos RD. Associação das doenças arterial periférica e cardiovascular na hipercolesterolemia familiar. Arq Bras Cardiol. 2014;103(2):118-23.

17. Horton JD, Cohen JC, Hobbs HH. PCSK9: a convertase that coordinates LDL catabolism. J Lipid Res. 2009;50(Suppl):S172-7.

18. Le Bras M, Roquilly A, Deckert V, Langhi C, Feuillet F, Sébille V, et al. Plasma PCSK9 is a late biomarker of severity in patients with severe trauma injury. Int J Clin Endocrinol Metab. 2013;98(4):732-6.

19. Souza GP, Pereira JAS, Judice WAS. Caracterização de inibidores sobre a atividade da pró-proteína convertase furina. Biochem Biotechnol Res. 2013;2(3):309-12.
20. Forti N, Salazar LA, Diament J, Giannini SD, Hirata MH, Hirata RD. Alterações genéticas e colesterolemia: recentes estudos brasileiros. Arq Bras Cardiol. 2003;80(5):565-71.

21. Botham KM, Mayes PA. Síntese, transporte e excreção do colesterol. In: Murray RK, Granner DK, Mayes PA, Rodwell VW. Harper: bioquímica. São Paulo: Atheneu; 2016. p. 266.

22. Beisiegel U, Weber W, Ihrke G, Herz, J, Stanley KK. The LDL-receptor-related protein, LRP, is an apolipoprotein E-binding protein. Nature. 1989;341(6238):162-4.

23. Maxwell KN, Fisher EA, Breslow JL. Overexpression of PCSK9 accelerates the degradation of the LDLR in a post-endoplasmic reticulum compartment. Proc Natl Acad Sci USA. 2005;102(6):2069-74.

24. Siqueira AF, Abdalla DS, Ferreira SR. LDL: from metabolic syndrome to instability of the atherosclerotic plaque. Arq Bras Endocrinol Metabol. 2006;50(2):334-43.

25. Stryer L. Biosynthesis of membrane lipids and steroids. In: Berg JM, Timoczkp JL, Stryer L. Biochemistry. New York: Macmillan; 1996. p. 697-8.

26. Genta MLND. Farmacocinética e captação tecidual do paclitaxel associado à nanoemulsão (LDE) em pacientes com neoplasias malignas do trato genital feminino [tese]. São Paulo: Universidade de São Paulo; 2006.

27. Elguindy A, Yacoub MH. The discovery of PCSK9 inhibitors: A tale of creativity and multifaceted translational research. Glob Cardiol Sci Pract. 2013;39(4):343-7.

28. Catapano AL, Papadopoulos N. The safety of therapeutic monoclonal antibodies: implications for cardiovascular disease and targeting the PCSK9 pathway. Atherosclerosis. 2013;228(1):18-28.

29. Cohen JC, Boerwinkle E, Mosley Jr TH, Hobbs HH. Sequence variations in PCSK9, low LDL, and protection against coronary heart disease. $\mathrm{N}$ Engl J Med. 2006;354(12):1264-72.

30. Giugliano RP, Desai NR, Kohli P, Rogers WJ, Somaratne R, Huang F, et al. Efficacy, safety, and tolerability of a monoclonal antibody to proprotein convertase subtilisin/kexin type 9 in combination with a statin in patients with hypercholesterolaemia (LAPLACE-TIMI 57): a randomised, placebo-controlled, dose-ranging, phase 2 study. Lancet. 2012;380(9858):2007-17.

31. Kohli P, Desai NR, Giugliano RP, Kim JB, Somaratne R, Huang F, et al. Design and rationale of the LAPLACETIMI 57 trial: a phase II, double-blind, placebo-controlled study of the efficacy and tolerability of a monoclonal antibody inhibitor of PCSK9 in subjects with hypercholesterolemia on background statin therapy. Clin Cardiol. 2012;35(7):385-91.

32. Cho L, Rocco M, Colquhoun D, Sullivan D, Rosenson RS, Dent $\mathrm{R}$, et al. Design and rationale of the GAUSS-2 study trial: a double-blind, ezetimibe-controlled phase 3 study of the efficacy and tolerability of evolocumab (AMG 145) in subjects with hypercholesterolemia who are intolerant of statin therapy. Clin Cardiol. 2014;37(3):131-9. 
33. Hirayama A, Honarpour N, Yoshida M, Yamashita S, Huang F, Wasserman SM, et al. Effects of evolocumab (AMG 145), a monoclonal antibody to PCSK9, in hypercholesterolemic, statin-treated Japanese patients at high cardiovascular risk. Circ J. 2014;78(5):1073-82.

34. Koren MJ, Lundqvist P, Bolognese M, Neutel JM, Monsalvo ML, Yang J, Bays H. Anti-PCSK9 monotherapy for hypercholesterolemia: the MENDEL-2 randomized, controlled phase III clinical trial of evolocumab. J Am Coll Cardiol. 2014;63(23):2531-40.

35. Stein EA, Giugliano RP, Koren MJ, Raal FJ, Roth EM, Weiss R, et al. Efficacy and safety of evolocumab (AMG 145), a fully human monoclonal antibody to PCSK9, in hyperlipidaemic patients on various background lipid therapies: pooled analysis of 1359 patients in four phase 2 trials. Eur Heart J Qual Care Clin Outcomes. 2014;35(33):2249-59.

36. Stroes E, Colquhoun D, Sullivan D, Civeira F, Rosenson RS, Watts GF, et al. Anti-PCSK9 antibody effectively lowers cholesterol in patients with statin intolerance: the GAUSS2 randomized, placebo-controlled phase 3 clinical trial of evolocumab. J Am Coll Cardiol. 2014;63(23):2541-8.

37. Sabatine MS, Giugliano RP, Wiviott SD, Raal FJ, Blom DJ, Robinson J, et al. Efficacy and safety of evolocumab in reducing lipids and cardiovascular events. N Engl J Med. 2015;372(16):1500-9.

38. Kiyosue A, Honarpour N, Kurtz C, Xue A, Wasserman SM, Hirayama A. A phase 3 study of evolocumab (AMG 145) in statin-treated Japanese patients at high cardiovascular risk. J Am Coll Cardiol. 2016;117(1):40-7.

\section{Como citar este artigo:}

Quim MHF et al. Atividade terapêutica do anticorpo monoclonal evolocumab contra proteínas PCSK9 na modulação do colesterol LDL: revisão. Rev. Aten. Saúde. 2017;15(52):72-77. 